\title{
Predictive Transmit Antenna Selection with Maximal Ratio Combining
}

\author{
Shiva Prakash \\ School of Computer Engineering \\ Block N4, Nanyang Avenue \\ Nanyang Technological University \\ Singapore 639798 \\ +6567906207 \\ shiv0008@ntu.edu.sg
}

\author{
Ian Mcloughlin \\ School of Computer Engineering \\ Block N4, Nanyang Avenue \\ Nanyang Technological University \\ Singapore 639798 \\ +6567906207 \\ mcloughlin@ntu.edu.sg
}

\begin{abstract}
Antenna selection has long been a pragmatic method for exploiting spatial diversity in wireless systems with lower complexity than space-time or MIMO coding, and potentially having reduced hardware cost due to the reduction in the number of $R F$ chains required. Whilst receive antenna selection is perhaps more common, transmit antenna selection also has several advantages, particularly for hardware-costly transmit schemes such as those requiring linearisation. However transmit antenna selection (TAS) requires either channel knowledge, or receiver knowledge at the transmitter, typically achieved using data transmission in the reverse direction, and this implies a delay between the channel being sampled and being acted upon. This outdated channel knowledge degrades system performance. In this paper, the degradation is determined, and related to the channel characteristics. A prediction scheme is then applied to mitigate against this degradation for the case of a $(2,1 ; 2)$ TAS system, where one of two transmit antenna is selected to communicate with two receive antennae employing maximal ratio combining.
\end{abstract}

\section{INTRODUCTION}

MIMO is often cited as a solution for achieving the high data rate demands of future wireless networks through increased spectral efficiency and link reliability [1]. Future MIMO systems are expected to further optimise performance by adapting to varying propagation and network conditions during operation - switching or adjusting parameters to perform better given the nature of current channel characteristics. These may include channel state information (CSI) or other measures of transmission environment, including low/high SNR levels or interference. Based on whether CSI is available at the transmitter, MIMO systems can be classified as either open or closed loop. A significant advantage of the traditional space time coding (STC) techniques is that they do not require any CSI knowledge at the transmitter, and are thus open loop. OSTBCs [2] [3] represent an important class of STC because they achieve full diversity while enjoying simple maximumlikelihood (ML) decoding. Considerable performance gains can be obtained in closed loop MIMO systems, by transmission on the Eigen-modes of the transmit antenna correlation matrix. For example, [4] proposes an optimal linear precoder that assumes knowledge of the transmit antenna correlations and improves the performance of STC by forcing transmission on the nonzero Eigen-modes of the transmit antenna correlation matrix. Although closed loop MIMO technology improves reliability and transmission rates, the improvement comes at the expense of higher hardware cost and increased feedback rates. The major constraints in implementing such systems are the cost of multiple transmit and receive radio chains, the form-factor limitation of multiple antennae for handheld devices and the complexity of the MIMO algorithms.

Transmit antenna selection (TAS) schemes employ partial CSI feedback to reduce transmitter complexity in closed loop systems by dynamically selecting a subset of available transmit antennae to maximise post processing SNR at the receiver. In this paper we analyse a TAS scheme having maximal ratio combining (MRC) at the receiver - an optimal combining scheme irrespective of channel fading statistics. MRC impacts complexity since it requires knowledge of all fading parameters and is suitable for most amplitude and phase modulated signals [5]. The TAS scheme requires only partial CSI, since only the index of the best antennae need be received at the transmitter end - accomplished either using a feedback link in frequency division duplex (FDD) systems, or by exploiting channel reciprocity in time division duplex (TDD) systems [6]. At high SNRs, such a TAS/MRC scheme achieves a full diversity order [7], as if all the transmit antennas were used, and also the scheme outperforms some of the complex spacetime codes of the same spectral efficiency.

Channel feedback delay is a major issue in TAS, significantly reducing antenna diversity, affecting the SEP of the TAS/MRC-based scheme. It is more significant in fast fading

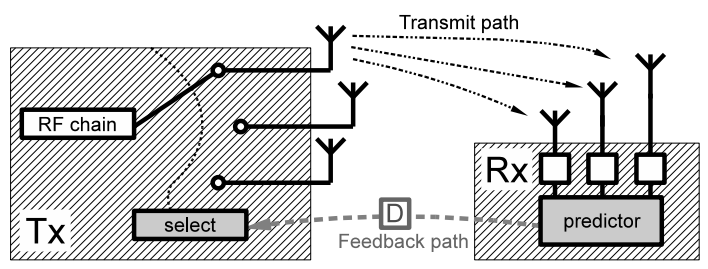

Fig. 1. Block diagram showing transmit antenna selection based upon information from a feedback path from MRC receive antennae and a predictor. 
channels where a delay associated with the return link might render any channel information completely outdated. In [7], the bit error rate (BER) of the TAS/MRC scheme was derived for binary phase shift keying (BPSK) in flat Rayleigh fading channels while in [8] the impact of CSI feedback delay over flat fading Rayleigh channels was considered.

To mitigate issues related to delay between channel measurement and switching, this paper considers a novel predictive-TAS scheme. The predictor exploits the time correlation of the channel. A power prediction is employed at the receiver which picks the best transmit antenna for transmission. The benefits of using predicted channel values for the TAS decisions are evaluated and compared with the delayed case. The paper is organized as follows. Section II presents the system structure and simulation models while Section III discusses the operation of TAS. Section IV discusses the investigations of TAS/predictor performance and their relevant operating characteristics, then Section V will conclude the paper.

\section{SYSTEM MODEL AND ASSUMPTIONS}

A flat fading MIMO channel with $N_{t}$ transmit and $N_{r}$ receive antennae is considered in a TAS/MRC system, shown in Fig. 1, where a single best transmitter $i$ is selected from $N_{t}$ candidates. At any time instant $k$ the received signal vector can be expressed as:

$$
\mathbf{y}(k)=\mathbf{h}_{i}(k) x(k)+\mathbf{z}(\mathbf{k})
$$

where $x(k)$ represents the uncoded symbol transmitted from the single selected antenna, $\mathbf{z}(k)$ is the AWGN vector with distribution $\mathcal{C N}\left(0, \sigma_{z}^{2} \mathbf{I}_{N_{r}}\right), \mathbf{h}_{i}(k)$ is a $N_{r} \times 1$ vector which is a column of the $N_{r} \times N_{t}$ channel matrix $\mathbf{H}(\mathbf{k})$. The entries of $\mathbf{H}$ are the fading coefficients $h_{i j}, 1 \leq i \leq N_{r}, 1 \leq j \leq N_{t}$ and are independent and identically distributed (i.i.d) Gaussian random variables $\mathcal{C N}\left(0, \sigma_{h^{2}}\right)$ that follow Jakes model [9] with Doppler spread $f_{d}$. A block flat or quasi-static fading model is considered with elements of $\mathbf{H}$ assumed to be constant over a frame and temporally correlated across blocks.

Channel estimates are assumed to be error-free at the receiver, so that noncausal channel smoothing with high accuracy can be performed using Wiener interpolator filters [10]. This assumption enables coherent detection at the receiver and is commonly adopted in such systems [11], [12]. Also an error-free feedback path is assumed: the use of powerful error control schemes could ensure this in practice. Due to decoding, ARQ, block buffering and other processes, these schemes introduce a time delay $\tau$ (expressed in symbol time). Even with perfect channel estimates at the receiver, the performance of an adaptive system such as TAS/MRC is degraded through delayed or outdated estimates of the channel at the transmitter. In this paper we analyse the BER performance impact of CSIdirected predictive TAS based on imperfect channel estimates, using MRC at the receiver.

\section{TAS PRINCIPLE}

\section{A. TAS without feedback delay}

The receiver picks the transmit antenna which offers the signal power $\gamma_{N_{t}}$ which maximises the post processing SNR at the output of the MRC receiver:

$$
\begin{gathered}
\gamma_{N_{t}}=\max _{1 \leq i \leq N_{t}}\left[\gamma_{i}\right] \\
\gamma_{i}=\frac{E_{s}}{N_{0}} \sum_{j=1}^{N_{r}}\left|h_{i j}\right|^{2}
\end{gathered}
$$

Index $i$ is fed back to the transmitter, through a feedback channel (shown in Fig. 1), switching after $\tau$ symbols.

\section{B. TAS with feedback delays (TASD)}

As discussed in Section I, channel feedback delay significantly reduces antenna diversity in fast fading channels. Large delays could render feedback information useless, effectively breaking the feedback loop. We know that the current channel $h(k)$ is related to its delayed version by:

$$
h_{\tau}(k)=\rho h(k)+\sqrt{1-\rho^{2}} z^{\prime}(k)
$$

where $\rho=J_{o}\left(2 \pi f_{d} \tau\right)$ is the correlated coefficient as per Jakes model, $\tau$ being the symbol delay, and $z^{\prime}(k)$ is AWGN with zero mean and unit variance.

The receiver computes the channel power corresponding to each antenna as per (3), picks the best antenna corresponding to maximum power gain as per (2) and then feeds back index $i$, reaching the transmitter after a time delay $n$. Due to this delay, the channel power at time $(n+k)$ is different from that at time $(k)$ thus affecting performance. For small values of the normalised feedback delay $f_{d} \tau<<1$, the BER degradation may be small, however at large delays, the system tends to behave like simple MRC with one transmit antenna because of the decrease in channel correlation. Fig. 2 shows the effect of increasing delays on the BER performance of a $(2,1 ; 2)$ TAS/MRC system (i.e. one which selects one of two transmit antenna and has two receive antennae). The error probability can be determined due to the delay [8], to reveal that TAS/MRC performance degrades considerably due to feedback delay.

\section{TAS with pilot-aided channel prediction (TASP)}

1) Channel prediction: Known pilot symbols are transmitted from each antennae in turn at different time slots into a fixed frame length $L_{f}$, and channel estimation for a frame of data is carried out independently for all channels. The entries of the channel matrix $\mathbf{H}$ are estimated as:

$$
\tilde{h}_{i j}(k)=h_{i j}(k)+v_{i j}(k)
$$

where $h_{i j}(k)$ is the true channel gain of the $k t h$ block and $\tilde{h}_{i j}(k)$ is the channel estimate while $v_{i j}(k)$ is the AWGN channel estimation error with distribution $\mathcal{C N}\left(0, \sigma_{v}^{2}\right)$, with $\sigma_{v}^{2}=N_{0} / E_{p}, E_{p}$ being the power of the pilot symbol. Thus the variance of the estimated channel amplitude given by $\sigma_{\tilde{h}}^{2}=$ 


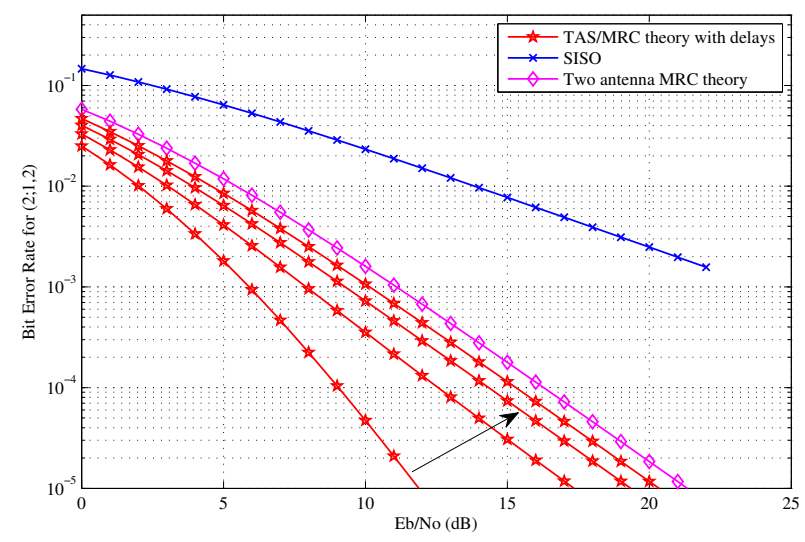

Fig. 2. Performance of TAS/MRC with feedback delay compared to SISO and MRC (the arrow indicates increasing feedback delay).

$\sigma_{h}^{2}+\sigma_{v}^{2}$. The CSI can be estimated using Weiner Hopf equations and the $n$ block ahead predicted channel can be written as $\hat{h}_{i j}(k+n)=\widetilde{\mathbf{h}}_{i n} w_{o p t}^{H}$ where $\widetilde{\mathbf{h}}_{i d}$ is the complex vector of estimated fading amplitudes corresponding to a prediction length

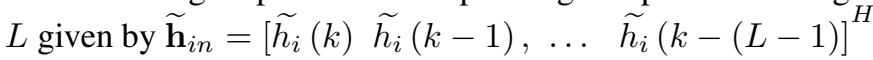
and $w_{o p t}$ is the complex coefficient vector given by $w_{o p t}=$ $R_{w}^{-1} r_{w}$ where $\left[R_{w}\right]_{i j}=J_{o}\left(2 \pi f_{d}|i-j| L_{f} T\right)+\sigma_{v}^{2}$ and $T$ is the symbol period, and $\left[r_{w}\right]_{i}=J_{o}\left(2 \pi f_{d}|n+i| L_{f} T\right)$. The correlation coefficient between the true and the predicted channel is given as $\hat{\rho}_{h \hat{h}}=\sqrt{ }\left[r_{w}^{H} R_{w}^{-1} r_{w}\right]$ which is bound by $0 \leq \hat{\rho}_{h \hat{h}} \leq 1$, a value of one meaning perfect prediction of channel estimates and zero meaning no correlation between predicted and estimated channel. The prediction error is given by $\epsilon(k)=h(k)-\hat{h}(k)$ with the minimum square error (MSE) being minimized when the optimal coefficient vector $w=w_{o p t}$ is used. Then the MSE is given by $\min _{w_{o p t}} \sigma_{\epsilon}^{2}=$ $\sigma_{h}^{2}-r_{w}^{H} R_{w}^{-1} r_{w}$ and is bound by $0 \leq \sigma_{\epsilon}^{2} \leq 1$. The true channel can then be written as:

$$
h(k)=\hat{h}(k)+\sqrt{\sigma_{h}^{2}-\hat{\rho}_{h \hat{h}}^{2}} n^{\prime}(k)
$$

where $n^{\prime}(k)$ is AWGN with zero mean and unit variance. The predicted channel amplitude is also a Gaussian random variable with variance $\sigma_{\hat{h}}^{2}=r_{w}^{H} R_{w}^{-1} r_{w}$.

\section{Prediction of channel power}

At instant $k$, the receiver selects a transmit antenna based on the predicted channel power given by:

$$
\widehat{\gamma}_{i}=\sum_{j=1}^{N_{r}}\left|\hat{h}_{i j}\right|^{2}
$$

and picks the antenna $i$ corresponding to maximum power gain just as we had seen for known channels in (2) and (3):

$$
\widehat{\gamma}_{\max }=\max _{1 \leq i \leq N_{t}}\left[\widehat{\gamma}_{i}\right]
$$

The average channel power gain is $E\left[\gamma_{i}\right]=N_{r} \sigma_{h}^{2}$, and the average predicted power gain is $E\left[\widehat{\gamma}_{i}\right]=N_{r} \sigma_{\hat{h}}^{2}=N_{r} r_{w}^{H} R_{w}^{-1} r_{w}$.
Note that the average value of the error $\epsilon_{p}=\gamma-\widehat{\gamma}$ while predicting power is not zero as was the case with simple channel amplitude prediction, it is in fact biased. It can be seen that $E\left[\epsilon_{p}\right]=N_{r}\left(\sigma_{h}^{2}-r_{w}^{H} R_{w}^{-1} r_{w}\right)$. Thus the power prediction is biased. The MSE for the biased predictor is $E\left[\sigma_{\epsilon_{p}}^{2}\right]=E\left[|\gamma-\widehat{\gamma}|^{2}\right]$. Using

$$
\begin{aligned}
& E\left[\gamma^{2}\right]=N_{r}\left(N_{r}+1\right) \sigma_{h}^{4} \\
& E\left[\hat{\gamma}^{2}\right]=N_{r}\left(N_{r}+1\right) \sigma_{\hat{h}}^{4} \\
& E[\gamma \hat{\gamma}]=N_{r}^{2} \sigma_{h}^{2} \sigma_{\hat{h}}^{2}+N_{r}\left|\hat{\rho}_{\tilde{h} \hat{h}}\right|^{4}
\end{aligned}
$$

The value of the MSE for the biased predictor can be determined. For perfect prediction, the error tends toward zero, and for no prediction the error will be equal to $\gamma^{2}$.

We can now proceed to determine channel SNR. First, let $X_{\max }$ be the true maximum SNR at time $(n+k)$ corresponding to the predicted maximum SNR $\hat{X}_{\max }$. Let $X_{i}$ represent the SNR at time $(n+k)$, then:

$$
X_{i}=\frac{E_{s}}{N_{0}} \gamma_{i}
$$

corresponding to the predicted power gain:

$$
\hat{X}_{i}=\frac{E_{s}}{N_{0}} \widehat{\gamma_{i}}
$$

for each antenna with their means as:

$$
\begin{gathered}
\bar{X}=E\left[X_{i}\right]=\frac{E_{s}}{N_{o}} N_{r} \sigma_{h}^{2}=r_{w}^{H} R_{w}^{-1} r_{w} \sigma_{h}^{2} \\
\overline{\hat{X}}=E[\hat{X}]=\frac{E_{s}}{N_{o}} N_{r} \sigma_{\hat{h}}^{2}
\end{gathered}
$$

Both are gamma distributed with PDFs, $X \sim G\left(N_{r}, \bar{x}\right)$ and $\hat{X} \sim G\left(N_{r}, \overline{\hat{x}}\right)$ where $\bar{x}=\frac{E_{s}}{N_{o}} \sigma_{h}^{2}$ and $\overline{\hat{x}}=\frac{E_{s}}{N_{o}} \sigma_{\hat{h}}^{2}$ being the shape factors of the gamma distributions also equal to the average SNR per symbol for the true and predicted channel respectively.

\section{E. Error probability analysis of a TAS/MRC with prediction}

In order to arrive at an expression for the the TASP/MRC symbol error probability, we first need to derive an expression for the PDF $f_{X \max }(x)$, where $X_{\max }$ is the maximum of the SNR $X_{i} s$ for all transmit antennas. The symbol error probability for the TASP/MRC $\left(N_{t}, 1 ; N_{r}\right)$ scheme can then be expressed as:

$$
B E R_{a v}=\frac{1}{\pi} \int_{0}^{\frac{\pi}{2}} M_{X}\left(-\frac{a^{2}}{\sin ^{2} \theta}\right) d \theta
$$

where $a$ is a constant that depends on the specific modulation scheme used [5] and $M_{X}$ is the moment generating function (MGF) of $f_{X \max }(x)$ given by:

$$
M(-s)=\int_{0}^{\infty} \exp (-s x) f_{X \max }(x) d x
$$

Using induced order statistics [13], the PDF of $X_{\max }$ can be determined: 


$$
\begin{aligned}
f_{X_{\max }}(x) & =\int_{0}^{\infty} f(x \mid \hat{x}) f_{\hat{X} \max }(\hat{x}) d \hat{x} \\
& =\int_{0}^{\infty} \frac{f(x, \hat{x})}{f_{\hat{X}}(\hat{x})} f_{\hat{X} \max }(\hat{x}) d \hat{x}
\end{aligned}
$$

where $f(x \mid \hat{x})$ is the PDF of $X$ conditioned on $\hat{X}$ and $f_{\hat{X}}(\hat{x})$ is the PDF of the predicted power $\hat{X}$ given by:

$$
f_{(\hat{X})}(\hat{x})=\frac{\hat{x}^{N_{r}-1}}{\overline{\hat{x}}^{N_{r}}\left(N_{r}-1\right) !} \exp \left(-\frac{\hat{x}}{\hat{\hat{x}}}\right)
$$

and $f_{\hat{X} \max }(\hat{x})$ is the PDF of $\hat{X}_{\max }$. In order to derive $f_{\hat{X} \text { max }}(\hat{x})$,we make use of the CDF of $\hat{X}$ given by

$$
F_{\hat{X}}(\hat{x})=1-\exp (-\hat{x} / \overline{\hat{x}}) \sum_{m=0}^{N_{r}-1}(1 / m !)(\hat{x} / \overline{\hat{x}})^{m}
$$

Again applying order statistics, the PDF $f_{\max }(\hat{X})$ can be given as:

$$
f_{\hat{X}_{\text {max }}}(\hat{x})=N_{t} f(\hat{x})[F(\hat{x})]^{N_{t}-1}
$$

Both $X$ and $\hat{X}$ are mutually correlated and their joint distribution is given by a bi-variate gamma distribution $G^{\prime} \sim$ $\left(N_{r}, \bar{x}, \overline{\hat{x}}, \rho_{p}\right)$ as follows:

$$
\begin{array}{r}
f_{X, \hat{X}}(x, \hat{x})=\left\{\frac{f(x) f(\hat{x})\left(N_{r}-1\right) !}{1-\rho_{p}}\left(\frac{\rho_{p} x \hat{x}}{\bar{x} \hat{x}}\right)^{-\left(N_{r}-1\right) / 2}\right. \\
I_{N_{r}-1}\left(\frac{2 \sqrt{\rho_{p}}}{\left(1-\rho_{p}\right)} \sqrt{\frac{x \hat{x}}{\bar{x}}}\right) \\
\left.\exp \left(-\frac{\rho_{p}}{1-\rho_{p}}\left(\frac{x}{\bar{x}}+\frac{\hat{x}}{\hat{x}}\right)\right)\right\}
\end{array}
$$

To proceed further, we express $\overline{\hat{X}}=r \bar{X}$ (since predicted SNR will be a fraction of the true SNR) where $0 \leq r \leq 1$. Substituting this in $(21,22,23)$, and expanding $f_{X}(x)$ (PDF of $\mathrm{X}$ ), which is similar to (22), we can write:

$$
\begin{array}{r}
f_{(X \mid \hat{X})}(x \mid \hat{x})=\left\{\left(\frac{r x}{\rho_{p} \hat{x}}\right)^{\left(N_{r}-1\right) / 2}\right. \\
I_{N_{r}-1}\left(\frac{2 \sqrt{\rho_{p} x \hat{x}}}{\left(1-\rho_{p}\right) \sqrt{r} \bar{x}}\right) \\
\left.\exp \left(-\frac{\rho_{p} \hat{x}+r x}{r\left(1-\rho_{p}\right) \bar{x}}\right) \cdot \frac{1}{\left(1-\rho_{p}\right) \bar{x}}\right\}
\end{array}
$$

where $I_{k}$ is the modified Bessel function of the first kind with order $N_{r}-1$ and $\rho_{p}$ is the correlation coefficient of the true and predicted SNRs, which is given by:

$$
\rho_{p}=\operatorname{Cov}(X, \hat{X})[\operatorname{Var}(X) \operatorname{Var}(\hat{X})]^{-0.5}
$$

Using $(9-11)$ and $(14,15)$ the value of $\rho_{p}$ can be calculated and shown to be equal to the square of channel correlation coefficient as $\rho_{h \hat{h}}^{2}$. Note that this equality occurs with the initial assumption that the coefficient vector $w_{o p t}$ is also used in channel power prediction calculations. Looking at (24), when $\rho_{p}$ goes to zero, the term in the exponential becomes independent of $\hat{x}$, and by expanding the Bessel function and clubbing it with the first term, we see that $f_{(X \mid \hat{X})}(x \mid \hat{x})$ becomes independent of $\hat{x}$ and becomes equal to $f_{X}(x)$ which is the PDF of X. This means that the SNR at time $(n+k)$, $X$ is independent of the predicted SNR $\hat{X}$, thus rendering TAS ineffective. The system behaves like a simple MRC system with a single transmit antenna $\left(1,1 ; N_{r}\right)$. By use of multinomial and binomial theorems, (22) can be expanded for use with (24) and the PDF derived as:

$$
\begin{gathered}
f_{X_{\max }}(x)=\frac{N_{t} ! x^{L_{r-1}} \exp \left(-\frac{x}{\left(1-\rho_{p}\right) x}\right)}{\left[\bar{x}\left(1-\rho_{p}\right)\right]^{N_{r}-1}\left[\left(N_{r}-1\right) !\right]^{2}} \\
\left\{\sum_{i=0}^{N_{t}-1}-1^{i}\left(\begin{array}{c}
N_{t}-1 \\
i
\end{array}\right) \sum_{j=0}^{i\left(N_{r}-1\right)}\left\{\left[\frac{\left(1-\rho_{p}\right)}{i\left(1-\rho_{p}\right)+1}\right]^{j+N_{r}}\right.\right. \\
\left.\left.\beta_{j i}\left(j+N_{r}-1\right) !_{1} F_{1}\left(j+N_{r} ; N_{r} ; \frac{\rho_{p} x}{\left[i\left(1-\rho_{p}\right)+1\right]\left(1-\rho_{p}\right) x}\right)\right\}\right\}
\end{gathered}
$$

where the coefficients $\beta_{j i}$ are recursively computed [14] as $\beta_{i 0}=1, \beta_{i 1}=1, \beta_{i i}=1 /((m-1) !)^{i}, \beta_{j i}=$ $\left.(1 / j) \sum_{l=1}^{C}(l(i+1)-k) / l !\right) \cdot \beta_{(j-i) i}$ with $C=\min (j, 1)$, $2 \leq k \leq-1 .{ }_{1} F_{1}(; ; ;)$ is the confluent hypergeometric function. Thus in (17) and thereby (16), the expression for the average BER can be computed using techniques as given in [5].

\section{INVESTIGATION}

\section{A. Influence of system parameters on channel correlation}

The correlation coefficient which governs the BER, in general depends on a number of parameters such as FIR predictor length, SNR, symbol rate, channel sampling frequency (location of pilot symbol), training length, Doppler frequency and feedback delay. At higher bit-rates, to limit the number of filter coefficients, it is beneficial to reduce pilot insertion frequency. This sub-sampling frequency (SSF) is usually kept at multiples of the Doppler frequency $\left(\mathrm{SSF} \geq 2 f_{d}\right.$ ). Having a relatively high over-sampling rate may mean clearer channel

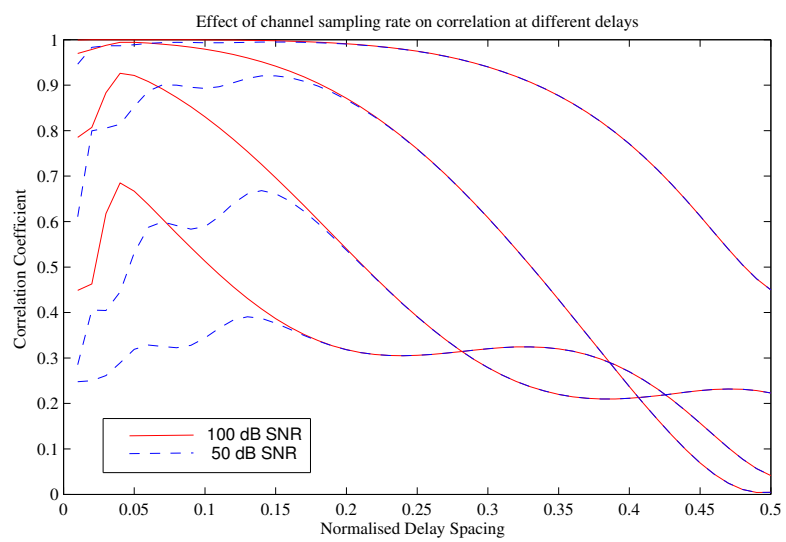

Fig. 3. Influence of filter tap spacing at different feedback delays $(0.2,0.4$, $0.6,0.8$ from top to bottom) on correlation, for fixed fifth order filters. 


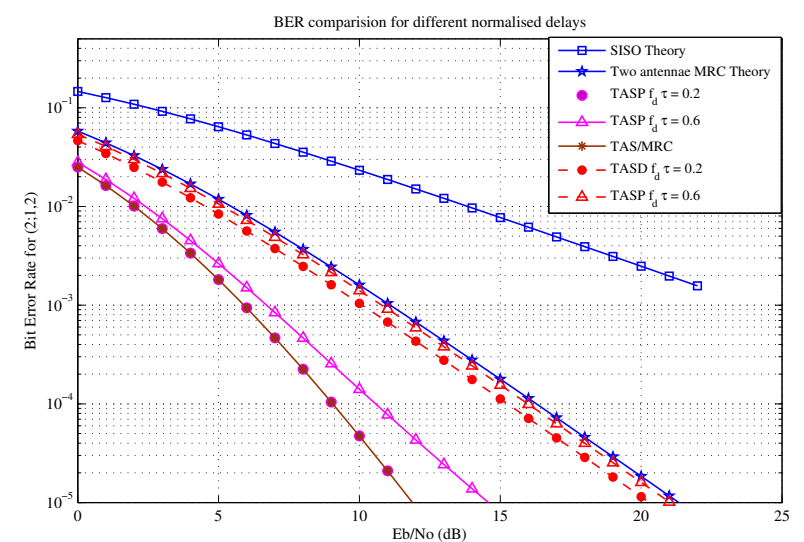

Fig. 4. Performance comparison of TAS/MRC with and without prediction for different delays at high $E_{p} / N_{0}$.

estimates but also poorer long range prediction with fixed number of filter coefficients and vice-versa.

In our simulations the symbol period is set as $T=1 \mu \mathrm{sec}$, with a Doppler of $100 \mathrm{~Hz}$, and using BPSK modulation. A 5-tap FIR predictor is used initially, although different filter lengths will be investigated later. To find the optimal sampling or adaptation rate, we must determine the optimal delay spacing at any given SNR and filter length. To find this, we plot channel correlation w.r.t to delay spacing at any given SNR and find the rate at which maximum correlation occurs. This is shown in Fig. 3. To observe full benefit of prediction, we maintain very high pilot to noise power ratio $E_{p} / N_{0}=100 \mathrm{~dB}$ at all SNR operating points, to have clear channel estimates for prediction. This is achievable in practice with a long training vector length, scaled with SNR.

Fig. 4, compares the performance of the TAS/MRC system with and without prediction for normalised feedback delays of 0.2 and 0.6. For a delay of 0.2 the performance of the TASP system is almost as good as the system without any feedback delay, since as seen in the figure the curves for TAS and TASP almost merge, and offers a gain of about $7.5 \mathrm{~dB}$ compared to the TASD at 10dB SNR. At 0.6 delay, the TASD behaves like an open-loop system or as good as a simple MRC system. The TASP system performs about $5 \mathrm{~dB}$ better, indicating that there is an increase in degradation rate w.r.t delay for the TASP case. This effect is seen more clearly in Fig. 5 which plots BER against normalised feedback delay for four different SNRs.

Even small amounts of delay in the TASD arrangement cause significant deterioration in BER. By contrast the predictive scheme (TASP) sustains performance almost unchanged out to around delays of 0.35 . For example at a carrier frequency of $900 \mathrm{MHz}$ at a walking speed of $1 \mathrm{~m} / \mathrm{s}$ and target BER of $10^{-4}$, in the TASD case, a time delay of not more than $3.3 \mathrm{~ms}$ can be tolerated. The TASP arrangement, however, can withstand a delay of upto $133 \mathrm{~ms}$. Similarly for vehicles moving at $22.2 \mathrm{~m} / \mathrm{s}(80 \mathrm{~km} / \mathrm{hr})$ the TASD case tolerates upto $149 \mu s$ and while the TASP can tolerate about $5.8 \mathrm{~ms}$ delay.

Fig. 5 also reveals that, for the TASD case, systems with

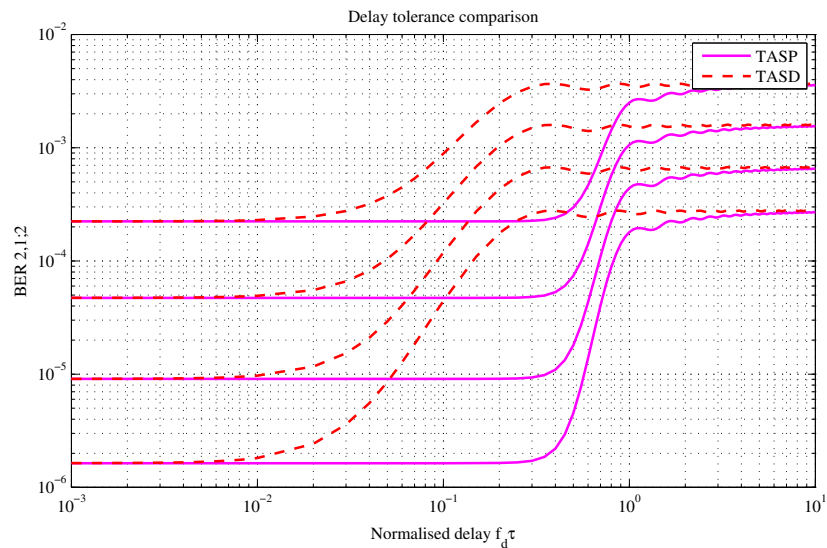

Fig. 5. Feedback delay tolerance comparison for SNRs of 8, 10, 12 and $14 \mathrm{~dB}$ (top to bottom) for the TASD and TASP arrangements at high $E_{p} / N_{0}$.

lower BER requirements are also more sensitive to feedback delay. For the TASP case, at a given SNR, the degradation is more pronounced at larger feedback delays than at smaller delays. This effect can be explained by looking at the power correlation coefficient $\rho_{p}$ in the BER equations. For the TASD case $\rho_{p}=J_{0}^{2}\left(2 p i f_{d} \tau\right)$ [9], which falls off rapidly for increasing values of $\tau$. By comparision the power correlation coefficient for the TASP case is equal to $r_{w}^{H} R_{w}^{-1} r_{w}$ which is a quadratic function having reduced slope at smaller values of delay compared to the coefficient in TASD case. The comparison of the correlation coefficient for both cases is plotted in Fig. 6, where the slope difference can be seen to be quite pronounced. For the TASP case the correlation is plotted for different system parameters and operating SNRs.

At a given SNR, normalised feedback delay and filter delay spacing, we can determine the dependency of the correlation and filter length with increasing number of pilot symbols for each antenna as $L_{t}$ is increased. This is shown in Fig. 7 for normalised delays of 0.2 and 0.6 at $12 \mathrm{~dB}$ SNR.

For quasi-static or block fading, we neglect the noise introduced by Doppler variance. Then the MSE or the variance

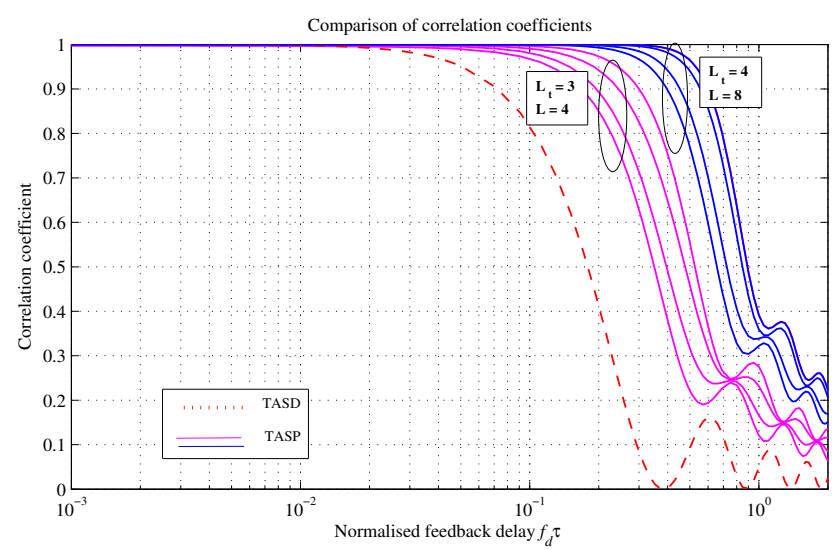

Fig. 6. Correlation coefficient comparison for TASD, and TASP for SNRS of $8,10,12,14 \mathrm{~dB}$ (bottom to top) with two different filter/training lengths. 

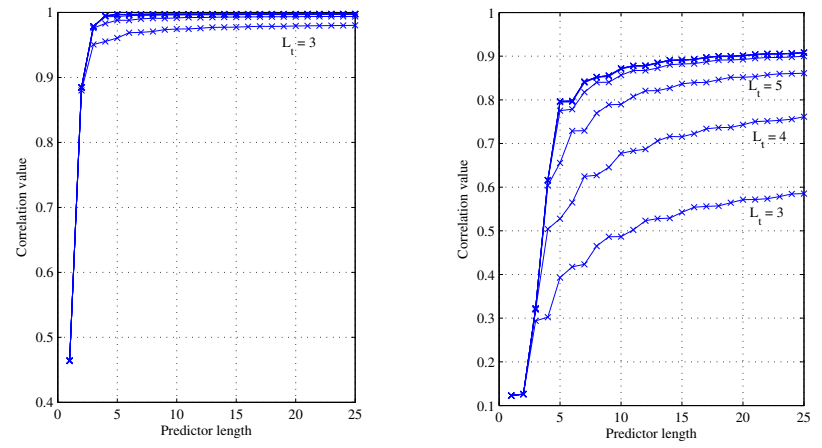

Fig. 7. Predictor length versus training length at $12 \mathrm{~dB}$ SNR for normalised delays of 0.2 (left) and 0.6 (right).

of the estimated channel will be $N_{0} / L_{t} E_{s}$ where $L_{t}$ is the number of pilot symbols for each transmit antenna (where each is transmitted round-robin), over the entire frame. The total number of pilot symbols for all antennae will be the training length given as $N_{t} L_{t}$. Thus $L_{t}$ will also influence the overall BER of the system. Obviously greater training length also decreases the duty cycle factor $\eta=1-N_{t} L_{t} / L_{d}$, affecting throughput.

For smaller delays, shorter training and prediction-filter lengths are able to assure sufficient correlation. However at increased delays, greater training length offer more correlation gain than increasing filter length, since clearer channel estimates offers greater prediction performance. This can be seen more clearly in Fig. 7, where two sets of curves (for delays of 0.2 and 0.6 ) for SNRS of 8,10,12,14 dB with different training and filter lengths are plotted. We can see that for a larger delay, the effect of changing training length is more pronounced. Maintaining the same parameters, and improving the operating SNR point, naturally increases correlation again because of better channel estimates. Depending on these system parameters, the correlation for the TASP system, increases or decreases at a fixed delay. At very small delays $\left(f_{d} \tau<=10^{-2}\right)$, when correlation is poor due to reduced training length, it may happen that TASD performs as well as, or even slightly better than TASP. Thus it maybe beneficial to turn off predication to save computation bandwidth under these circumstances. Depending on the required output BER, we can determine the optimal values of predictor order and $L_{t}$ from such graphs. For example, given an operating point at $10 \mathrm{~dB}$ and target BER of $10^{-3}$, we first obtain the required channel correlation from the BER expression to sustain the error rate. Next we determine the optimal training and filter lengths to achieve this correlation value. For example, if we require a 0.85 correlation to achieve an error rate of $10^{-3}$ at $10 \mathrm{~dB}$, then from Fig. 6, a training and filter length of 3 and 4 could be used to achieve this correlation. Further for these values, the system will be able of tolerating a normalised feedback delay of up to 0.2 .

\section{Conclusion}

This paper has explored the issue of transmit antenna selection, based upon feedback from a MRC receiver. In general, a system with two receive antennae employing MRC, and two transmit antenna, one of which is selected for transmission of an entire data frame, was the system model. TAS was evaluated in the presence of switching delays, and shown to degrade performance for even quite small delays.

To mitigate against the performance degradation caused by a time delay between channel evaluation and transmitter switching, a predictor was developed to utilise past CSI to predict the best transmitter for the current data frame. This has been shown to be capable of alleviating much of the performance loss associated with outdated transmitter selection knowledge, even at delays which would cause non-predictive TAS to be completely ineffective. The inter-relation between systems parameters such as predictor and training length and operating parameters such as SNR have also been explored, and the critical importance of the channel correlation coefficient noted. Since the prediction scheme is relatively low in complexity, it would be a viable choice for TAS systems experiencing medium to severe feedback delays.

\section{REFERENCES}

[1] A. Paulraj, D. Gore, R. Nabar, and H. Bolcskei, "An overview of MIMO communications-a key to gigabit wireless," Proceedings of the IEEE, vol. 92, no. 2, pp. 198-218, 2004.

[2] V. Tarokh, H. Jafarkhani, and A. Calderbank, "Space-time block codes from orthogonal designs," IEEE Transactions on Information Theory, vol. 45 , no. 5 , pp. 1456-1467, 1999.

[3] M. Jankiraman, Space-time codes and MIMO systems. Artech House Publishers, 2004.

[4] H. Sampath, A. Paulraj, I. Inc, and C. San Jose, "Linear precoding for space-time coded systems with known fading correlations," IEEE Communications Letters, vol. 6, no. 6, pp. 239-241, 2002.

[5] M. Simon and M. Alouini, Digital communication over fading channels. Wiley-Interscience, 2005.

[6] A. Molisch, M. Win, Y. Choi, and J. Winters, "Capacity of MIMO systems with antenna selection," IEEE Transactions on Wireless Communications, vol. 4, no. 4, pp. 1759-1772, 2005.

[7] Z. Chen, J. Yuan, and B. Vucetic, "Analysis of transmit antenna selection/maximal-ratio combining in Rayleigh fading channels," IEEE Transactions on Vehicular Technology, vol. 54, no. 4, pp. 1312-1321, 2005.

[8] J. Tang and X. Zhang, "Error probability analysis of TAS/MRC-based scheme for wireless networks [point-to-point link example]," in 2005 IEEE Wireless Communications and Networking Conference, vol. 2, 2005.

[9] W. Jakes, Microwave mobile communications. Wiley-IEEE Press, 1994.

[10] G. Oien, H. Holm, and K. Hole, "Impact of channel prediction on adaptive coded modulation performance in Rayleigh fading," IEEE Transactions on Vehicular Technology, vol. 53, no. 3, pp. 758-769, 2004.

[11] M. Alouini and A. Goldsmith, "Adaptive modulation over Nakagami fading channels," Wireless Personal Communications, vol. 13, no. 1, pp. 119-143, 2000.

[12] S. Zhou and G. Giannakis, "How accurate channel prediction needs to be for transmit-beamforming with adaptive modulation over Rayleigh MIMO channels?" IEEE Transactions on Wireless Communications, vol. 3, no. 4, pp. 1285-1294, 2004.

[13] H. David and H. Nagaraja, Order statistics. Wiley-Interscience, 2004

[14] M. Alouini and M. Simon, "Performance of coherent receivers with hybrid SC/MRC overNakagami-m fading channels," IEEE Transactions on Vehicular Technology, vol. 48, no. 4, pp. 1155-1164, 1999. 